\title{
Star formation histories of resolved galaxies
}

\author{
Monica Tosi \\ INAF - Osservatorio Astronomico di Bologna \\ Via Ranzani 1, I-40127, Bologna, Italy \\ email: monica.tosi@oabo.inaf .it
}

\begin{abstract}
The colour-magnitude diagrams of resolved stellar populations are the best tool to study the star formation histories of the host galactic regions. In this review the method to derive star formation histories by means of synthetic colour-magnitude diagrams is briefly outlined, and the results of its application to resolved galaxies of various morphological types are summarized. It is shown that all the galaxies studied so far were already forming stars at the lookback time reached by the observational data, independently of morphological type and metallicity. Early-type galaxies have formed stars predominantly, but in several cases not exclusively, at the earliest epochs. All the other galaxies appear to have experienced rather continuous star formation activities throughout their lifetimes, although with significant rate variations and, sometimes, short quiescent phases.
\end{abstract}

Keywords. Hertzsprung-Russell diagram, galaxies: stellar content, galaxies: individual (M31, M33, LMC, SMC, Leo A), Local Group, Magellanic Clouds, galaxies: evolution

\section{Introduction}

Two complementary approaches are necessary to understand galaxy evolution: on the one hand, we need to develop theoretical models for galaxy formation, chemical and dynamical evolution, and on the other hand, we need to collect as many and as accurate as possible observational data to constrain such models. In particular, we need to know the masses, chemical abundances and kinematics of the various galactic components, namely gas, stars and dark matter; we need to know the star formation history (SFH), the initial mass function (IMF), etc. In this review our current knowledge of the SFHs, as derived from the colour-magnitude diagrams (CMDs) of their resolved stellar populations, is summarized.

Resolved stellar populations are the best tracers of the SFH of a galactic region, and their CMD the best tool to exploit the tracers. This is due to the well known circumstance that the location of any individual star in a CMD is uniquely related to its mass, age and chemical composition. From the CMD we can thus disentangle directly these evolution parameters. In the case of simple stellar populations, i.e. coeval stars with the same chemical composition, isochrone fitting is the most frequently used method to infer the system age. In the case of galaxies, with rather complicated mixtures of different stellar generations, the age determination is less straightforward, but their CMDs remain the best means to derive the SFH.

\section{CMD synthesis and star formation histories}

To visualize how the SFH affects the CMD morphology, a few representative cases are displayed in Fig. 1. The six panels of the Figure show the effect of different SFHs on the synthetic CMD of a hypothetical galactic region with number of resolved individual stars, photometric errors, blending and incompleteness factors typical of a region in the 


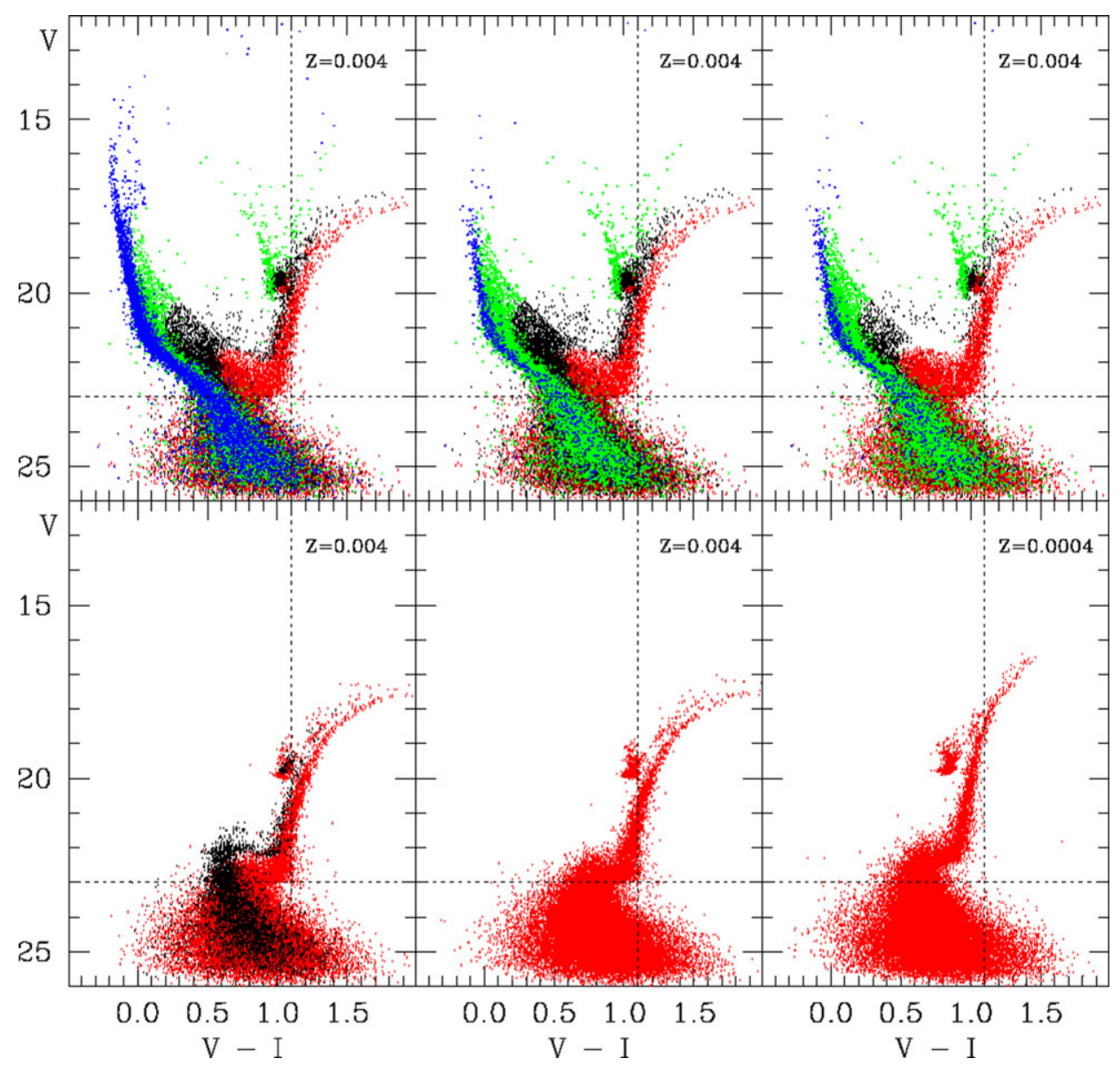

Figure 1. The effect of the SFH on the theoretical CMD of a hypothetical galactic region with $(\mathrm{m}-\mathrm{M})_{0}=19, \mathrm{E}(\mathrm{B}-\mathrm{V})=0.08$, and with the photometric errors and incompleteness typical of HST/WFPC2 photometry. All the shown synthetic CMDs contain 50000 stars and are based on the Padova models (Fagotto et al. 1994a, Fagotto et al. 1994b) with the labeled metallicities. Top-central panel: the case of a SFR constant from $13 \mathrm{Gyr}$ ago to the present epoch. Top-left panel: the effect of adding a burst 10 times stronger in the last $20 \mathrm{Myr}$ to the constant SFR. The CMD has a much brighter and thicker blue plume. Top-right panel: same constant SFR as in the first case, but with a quiescence interval between 3 and 2 Gyrs ago; a gap appears in the CMD region corresponding to stars 2-3 Gyr old, which are completely missing. Bottom-central panel: SF activity only between 13 and $10 \mathrm{Gyr}$ ago with $\mathrm{Z}=0.004$. Bottom-right panel: SF activity only between 13 and 10 Gyr ago with $\mathrm{Z}=0.0004$ : notice how colour and luminosity of turnoff, subgiant and red giant branches differ from the previous case. Bottom-left panel: SF activity between 13 and 11 Gyr ago, followed by a second episode of activity between 5 and 4 Gyr ago: a gap separates the two populations in the CMD, but less evident than in the top-right panel case, when the quiescent interval was more recent.

SMC imaged with HST/WFPC2. If the SFH of the studied region has been one of the following six cases, then, according to stellar evolution models, the CMD of its stars is one of those shown in Fig. 1. The top three panels show examples of CMDs typical of late-type galaxies, with ongoing or recent star formation activity. If the star formation rate (SFR) has been constant for all the galaxy lifetime, the CMD of the region is expected to have the morphology of the top-central panel, with a prominent blue plume mostly populated 
by main-sequence (MS) stars and an equally prominent red plume resulting from the overposition of increasingly bright and massive stars in the red giant branch (RGB), asymptotic giant branch (AGB) and red supergiant phases. At intermediate colours, for decreasing brightness, stars in the blue loops and subgiant phases are visible, as well stars at the oldest MS turnoff (MSTO) and on the faint MS of low mass stars. Stars of all ages are present, from those as old as the Hubble time to the brightest ones a few tens Myr old.

If we leave the SFH unchanged except for the addition of a burst ten times stronger concentrated in the last $20 \mathrm{Myr}$, the CMD (top-left panel) has a much brighter and more populated blue plume, now containing also stars a few Myr old. In the top-right panel the same constant SFR as in the first case is assumed, but with a quiescent interval between 3 and 2 Gyrs ago: a gap is clearly visible in the CMD region corresponding to the age of the missing stars.

The three bottom panels of Fig. 1 show CMDs typical of early-type galaxies, whose SF activity is concentrated at the earliest epochs. If only one SF episode has occurred from 13 to $10 \mathrm{Gyr}$ ago, with a constant metallicity $\mathrm{Z}=0.004$ as in the top panel cases, the resulting CMD is shown in the bottom-central panel. If the SF has occurred at the same epoch, but with a metallicity ten times lower, the evolutionary phases in the resulting CMD (bottom-right panel) have colours and luminosities quite different from the previous case. Finally, the bottom-left panel shows the case of two bursts, the first from 13 to 11 Gyr ago and the second from 5 to 4 Gyr ago. The gap corresponding to the quiescent interval is evident in the CMD, although not as much as the more recent gap of the top-right panel.

The tight dependence of the CMD morphology on the SFH is the cornerstone of the synthetic CMD method, which consists in comparing the observational CMD of a galactic region with synthetic CMDs, such as those of Fig. 1, created via Monte Carlo extractions on stellar evolution tracks or isochrones for a variety of SFHs, IMFs, binary fractions and age-metallicity relations (see e.g. Tosi et al. 1991, Tolstoy 1996, Greggio et al. 1998, Aparicio \& Gallart 2004 for detailed descriptions of different procedures). The synthetic CMDs take into account the number of stars, photometric errors, incompleteness and blending factors of the observational CMD (or portions of it). Hence, a combination of assumed parameters is acceptable only if the resulting synthetic CMD reproduces all the features of the observational one: morphology, colours, luminosity functions, number of stars in specific evolutionary phases. The method does not provide unique solutions, but significantly reduces the possible SFH scenarios.

At its first applications to photometric data from ground-based, moderate size telescopes the synthetic CMD method demonstrated its power, showing that even in tiny galaxies such as Local Group dwarf irregulars (dIrrs) the SFH varies from one region to the other and that their star formation regime is rather continuous, with long episodes of moderate activity, separated by short quiescent intervals (the so-called gasping regime, Ferraro et al. 1989, Tosi et al. 1991, Marconi et al. 1995, Gallart et al. 1996, Tolstoy 1996) and not the bursting regime (short episodes of strong SF activity separated by long quiescent phases) that most people attributed to late-type dwarfs at the time.

When the first non-aberrated images were acquired with HST, the impressive improvement in the achievable photometric resolution and depth, and the corresponding quantum leap in the quality of the CMDs, triggered a worldwide burst of interest in the derivation of the SFHs of nearby galaxies and in the synthetic CMD method. Many people developed their own procedures and to date a large fraction of Local Group galaxies have had the SFH of at least some of their regions derived with the synthetic CMD method. Nowadays, in LG galaxies it is possible to resolve individual stars down to faint/old objects 
in all galactic regions and we can thus infer the SFHs over long lookback times $\tau$ (up to the Hubble time), with an average time resolution around $(0.1-0.2) \tau$.
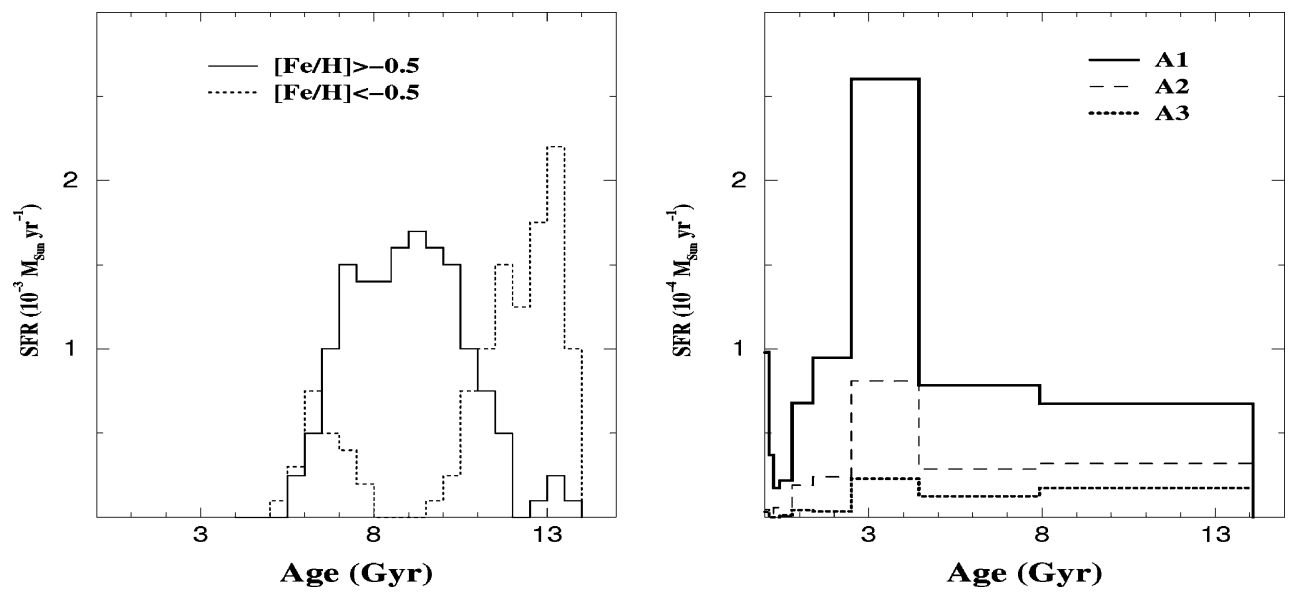

Figure 2. SFH from CMDs in (small) regions of the two LG external spirals. The left-hand panel shows the SFH of first HST/ACS field of M31 studied by Brown (2006), who divided the stars according to their metallicity. The right-hand panel shows the SFHs of the three HST/ACS fields of M33, A1, A2 and A3, studied by Barker et al. (2007).

\section{Star formation histories of Local Group galaxies}

The current census of LG galaxies with SFH derived in some of their regions with the synthetic CMD method is impressive. SFHs have been inferred from CMDs of both the two external spiral galaxies, M31 and M33, the two Magellanic Clouds, LMC and SMC, a dozen dIrrs, 5 transition type dwarfs and about 20 early-type dwarfs (dwarf spheroidals, dSphs, and dwarf ellipticals, dEs).

In M31, long HST/ACS exposures have allowed Brown et al.(2008) to resolve stars fainter than the oldest MSTO in three regions and derive their SFH back to the earliest epochs. They find a fairly continuous activity through the whole lifetime of Andromeda. The SFH in the first M31 field studied by Brown is shown in the left-hand panel of Fig. 2: if the SFH resulting from both metal poor and rich stars is considered, it turns out to have been rather constant. In M33, HST/ACS imaging has allowed Barker et al. (2007) to study three different regions, again resolving their oldest stars. The resulting $\mathrm{SFH}$ (right-hand panel of Fig. 2) clearly differs from one region to the other and shows significant bumps and gasps over a rather continuous mode. In all the three regions was the SF activity already in place a Hubble time ago. It is apparent that the SF activity in the M31 field has been both stronger and more constant than in M33.

The SFHs of several regions of the Magellanic Clouds have been studied by a number of authors, both from space and from ground (e.g. Holtzman et al. 1999, Dolphin et al. 2001, Smecker-Hane et al. 2002, Harris \& Zaritsky 2004, Chiosi et al. 2006, Noel et al. 2007, Cignoni et al. 2009). Their proximity makes the oldest stars visible also from ground, with the advantage of fields of view larger than those of the HST cameras. Harris \& Zaritsky (2004) even covered the whole SMC. On the other hand, the exquisite spatial resolution of HST is necessary to resolve and study the fainter stars in crowded regions, such as those of the star forming clusters. While stars at the oldest MSTOs and subgiant branches are the unique means to firmly establish the SFH at the earliest epochs, pre-MS 

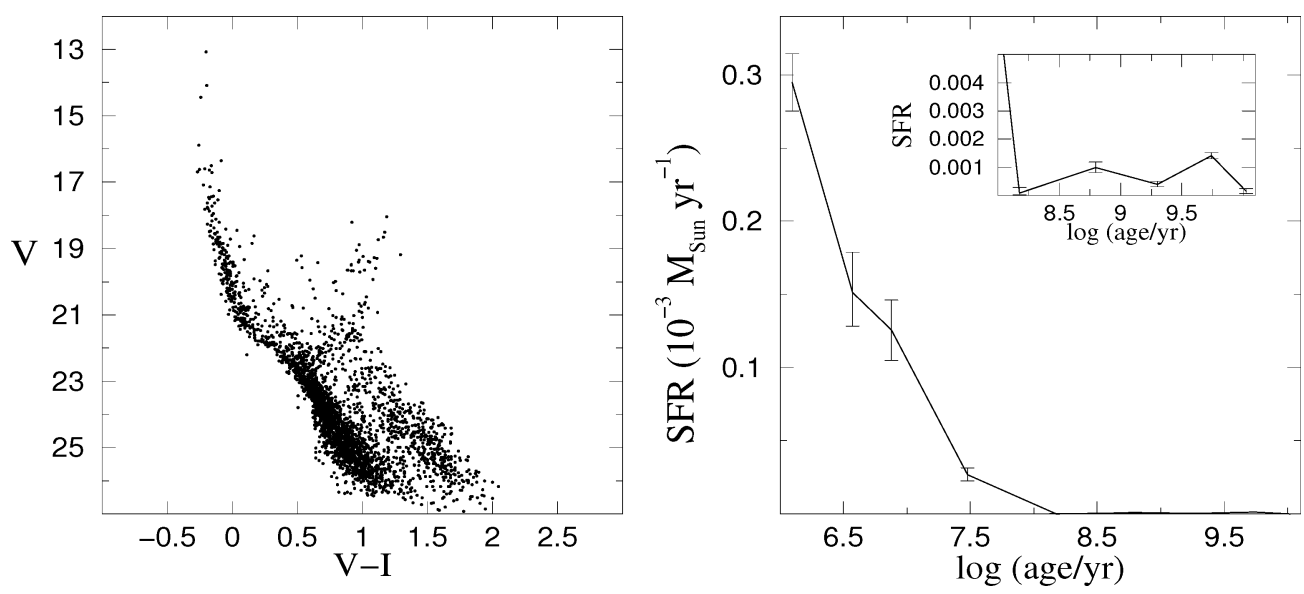

Figure 3. Left-hand panel: CMD of the HST/ACS field around the young cluster NGC 602 in the SMC. The red sequence of pre-MS stars is easily recognizable parallel to the lower MS. The bright blue plume contains the young cluster stars while the lower MS is only populated by field stars, since the cluster stars with mass below $\sim 1 M_{\odot}$ haven't yet had time to reach it. Right-hand panel: corresponding SFH as derived with the synthetic CMD method (Cignoni et al. 2009). The oldest part of the SFH is zoomed-in in the upper right inset.

stars are precious tools to study the details of the most recent $\mathrm{SFH}$ in terms of time and space behaviour (Cignoni et al. 2009). The SMC regions of intense recent star formation can provide key information on the star formation mechanisms in environments with metallicity much lower than in any Galactic star forming region. Fig. 3 shows the CMD of the young cluster NGC 602 in the Wing of the SMC, observed with HST/ACS. Both very young stars (either on the upper MS or still on the pre-MS) and old stars are found. The SFH of the cluster and the surrounding field is also shown, revealing that the cluster has formed most of its stars around 2.5 Myr ago, while the surrounding field has formed stars continuously since the earliest epochs. All the studies on the MC fields have found that the SFHs of their different regions differ from one another in the details (e.g. epoch of activity peaks, enrichment history, etc.) but are always characterized by a gasping regime, i.e. a rather continuous activity since the earliest epochs, but with significant peaks and gasps. In the LMC a clear difference has been found between the SFH of field stars and of star clusters, the latter showing a quiescence phase, several Gyr long, absent in the field.

Dwarf irregulars were the first systems to which synthetic CMD analyses were applied. HST has had a large impact on studies of these systems. The high spatial resolution of its cameras have allowed Dohm-Palmer et al. (1998) and Dohm-Palmer et al. (2002) to spatially resolve and measure the SF activity over the last $0.5 \mathrm{Gyr}$ in all the sub-regions of the dIrrs Gr8 and Sextans A, close to the borders of the LG. The resulting space and time distribution of the SF, with lightening and fading of adjacent cells, is intriguingly reminiscent of the predictions of the stochastic self-propagating SF theory proposed by Seiden, Schulman, \& Gerola(1979) 30 years ago. The HST/ACS is currently providing the deepest and tighter CMDs of dIrrs ever obtained, likely to remain unequaled for a very long time. These spectacular CMDs reach well below the oldest MSTO and allow the derivation of the SFH back to a Hubble time ago. The first of such impressive studies is that of Leo A (Cole et al. 2007), whose CMD and SFH are plotted in Fig. 4. In Leo A the star formation activity was present, although quite low, at the earliest epochs, and 

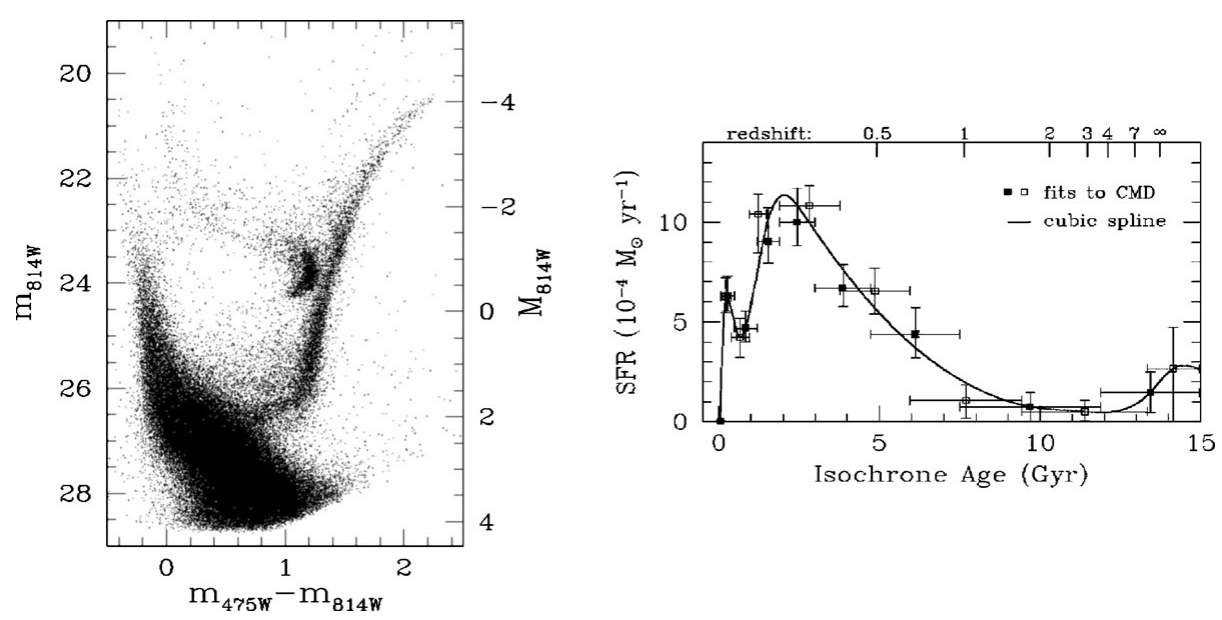

Figure 4. CMD and SFH of Leo A as derived by Cole et al. (2007) from HST/ACS data. Notice the impressive depth and tightness of the CMD, allowing to infer the SFH even at the earliest epochs.

$90 \%$ of the activity occurred in the last $8 \mathrm{Gyr}$, with the main peak around 2 Gyr ago and a secondary peak a few hundreds Myr ago. Once again, the results obtained so far show that dIrrs experience a rather continuous star formation since the earliest epochs, but with significant peaks and gasps.

To find SFHs peaked at earlier epochs one needs to look at early-type dwarfs: dEs, dSphs and even transition-type dwarfs clearly underwent their major activity around or beyond 10 Gyr ago. The latter also have significant activity at recent epochs (e.g. Young et al. 2007). The former have few (or no) episodes of moderate activity in the last several Gyrs (e.g. Smecker-Hane et al. 1996, Hurley-Keller et al. 1998, Hernandez, Gilmore \& Valls-Gabaud 2000, Dolphin 2002, Dolphin et al. 2005).

The beautiful CMDs from Carme Gallart's L-CID HST program on 6 dwarfs of different type (two dIrrs, two dSphs and two transition type, see Hidalgo et al. this volume) promise to provide SFHs of unprecedented time resolution for external galaxies. Another interesting project is trying to treat homogeneously all the LG galaxies observed with the HST/WFPC2, deriving the CMDs of their resolved populations in a self-consistent way (Holtzman, Afonso \& Dolphin 2006) and the corresponding SFH with the same technique and assumptions (Dolphin et al. in preparation, see also Dolphin et al. 2005). Homogeneous data sets and analyses are valuable to obtain a uniform overview of the properties of the different galaxies in the LG.

\section{Star formation histories of galaxies outside the Local Group}

In galaxies beyond the LG, distance makes crowding more severe, and even HST cannot resolve stars as faint as the MSTO of old populations. The higher the distance, the worse the crowding conditions, and the shorter the lookback time $\tau$ reachable even with the deepest, highest resolution photometry. Depending on distance and intrinsic crowding, the reachable $\tau$ in galaxies more than $1 \mathrm{Mpc}$ away ranges from several Gyrs (in the best cases, when the RGB or even the HB are clearly identified), to several hundreds Myr (when AGB stars are recognized), to a few tens Myr (when only the brightest supergiants are resolved). 


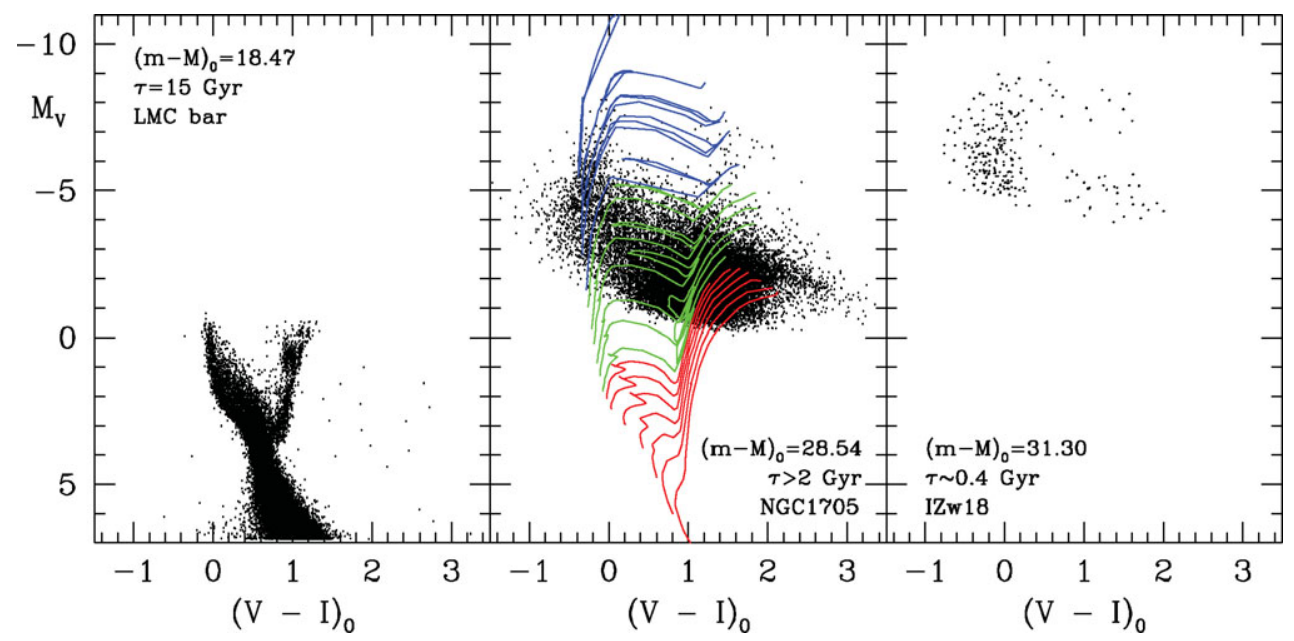

Figure 5. Effect of distance on the resolution of individual stars and on the corresponding lookback time $\tau$ for the SFH. CMD in absolute magnitude and colour of systems observed with the HST/WFPC2 and analysed with the same techniques, but at different distances; from left to right: $50 \mathrm{Kpc}$ (LMC bar), $5.1 \mathrm{Mpc}$ (NGC1705) and $18 \mathrm{Mpc}$ (IZw18). The central panel also shows stellar evolution tracks from Fagotto et al. (1994b) for reference: red lines refer to low-mass stars, green lines to intermediate mass stars, and blue lines to massive stars.

The effect of distance on the possibility of resolving individual stars, and therefore on the reachable $\tau$, is shown in Fig. 5, where the CMDs obtained from WFPC2 photometry of three late-type galaxies are shown: the LMC bar (Smecker-Hane et al. 2002), with a distance modulus of $18.47(50 \mathrm{kpc})$ and a CMD reaching several mags below the old MSTO; NGC1705 (Tosi et al. 2001), with distance modulus 28.54 (5.1 Mpc) and a CMD reaching a few mags below the tip of the RGB; and IZw18 (Aloisi, Tosi \& Greggio 1999), with the new distance modulus $31.3(18 \mathrm{Mpc})$ derived by Aloisi et al. (2007). Notice that the latter modulus is inferred from the periods and luminosities of a few classical Cepheids measured from HST/ACS data which also allowed us to reach the RGB, but the WFPC2 data shown in Fig. 5 allow to reach only the AGB. The CMD obtained from the ACS is shown in Fig. 6.

Since the Local Group doesn't host all types of galaxies, with the notable and unfortunate absence of both the most and the least evolved ones (ellipticals and Blue Compact Dwarfs, BCDs, respectively), a few people have tackled the challenging task of deriving the SFH of more distant galaxies. In spite of the larger uncertainties and the shorter lookback time, these studies have led to quite interesting results, which wouldn't have been possible without HST.

First of all, all the galaxies, including BCDs, where individual stars have been resolved by HST, and the SFH has been derived with the synthetic CMD method, have turned out to be already active at the lookback time reached by the photometry (see e.g. Lynds et al. 1998, Aloisi, et al. 1999, Schulte-Ladbeck et al. 2000, Schulte-Ladbeck et al. 2001, Annibali et al. 2003, Rejkuba, Greggio \& Zoccali 2004, Vallenari Schmidtobreik \& Bomans 2005). None of them appears to be experiencing now its first star formation activity, including the most metal poor ones, such as SBS1415 and IZw18 (see Aloisi et al. 2005 and Aloisi et al. 2007). Fig. 7 sketches the SFHs derived by various authors for some of the starburst dwarfs studied so far and one low surface brightness dwarf, UGC 5889. The lookback time is indicated and in all cases stars with that age were detected. 
All the late-type dwarfs of Fig. 7 present a recent SF burst, which is what let people discover them in spite of the distance, and none of them exhibits long quiescent phases within the reached $\tau$. It is interesting to notice that the SFH of the low surface brightness dwarf UGC 5889 (Vallenari et al. 2005) is also qualitatively similar to that of starburst dwarfs, except that the SFR is definitely moderate. In all the shown galaxies the strongest $\mathrm{SF}$ episodes are overimposed over a rather continuous, moderate SF, already in place at the $\tau$ reached by the photometry. Indeed, no one has ever found yet a galaxy without stars as old as $\tau$ from the CMDs of the resolved populations.

The SF rate differs significantly from one galaxy to the other. The two most powerful bursts measured so far are the recent ones in NGC 1705 and NGC 1569, with SFR per unit area a factor 10-100 higher than in the other starbursting dwarfs studied through their CMDs. Intriguingly, the strongest of all is not a BCD, but the dwarf irregular NGC 1569, suggesting that the morphological classification of these faint small galaxies was possibly affected by their distance and the capability of resolving their shape with ground-based small telescopes, at the time of their discovery. Had it been at $20 \mathrm{Mpc}$, NGC 1569 would have probably been classified as a BCD.

\section{Discussion}

From the comparison of the SFHs of starburst dwarfs with those of Local Group dwarf irregulars, one can see that in both cases the SF regime is rather continuous (gasping), with two main differences: starburst dwarfs always have the strongest SF episode at recent epochs, while the current SF activity of local irregulars is not necessarily the highest peak. Leo A, with the main SF peak a few Gyr ago (Fig. 4) is quite typical. On the other hand, it is interesting to notice that the SF of the SMC region around the very young cluster NGC 602 (Fig. 3), host of HII regions, shows time distribution and current rate per unit area similar to those (Fig. 7) of starburst dwarfs (once called extragalactic HII regions). The former however involves a small area, corresponding to a tiny fraction of the SMC, while the latter are global behaviours, referring to the whole galaxy.

By comparing with each other the SFHs derived from the CMDs of (few, small) regions of the LG spirals (Fig. 2), one is tempted to speculate over a possible dependence of the SFHs on their morphological type and luminosity class. M31 (SA b I-II) seems to have had very continuous, almost constant $\mathrm{SF}$, since the earliest epochs. The solar neighbourhood

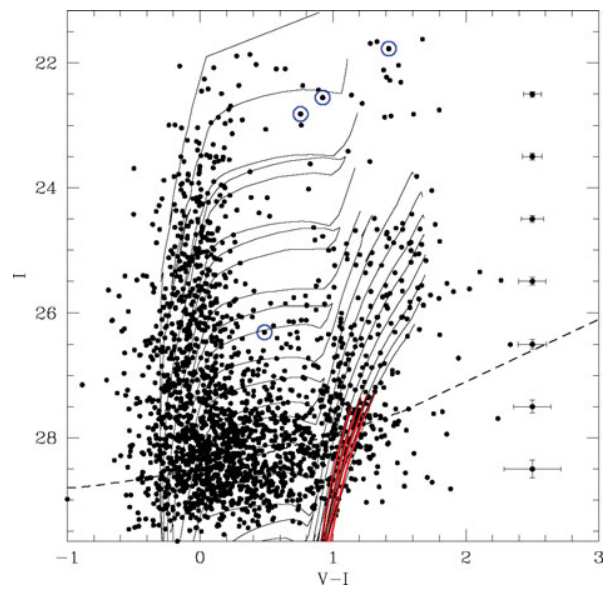

Figure 6. CMD of IZw18, obtained from HST/ACS imaging (Aloisi et al. 2007). Overimposed are the $\mathrm{Z}=0.0004$ isochrones by Bertelli et al. (1994) with the RGB in red. Also shown is the average position of the 4 classical Cepheids with reliable light-curves obtained from these data. 

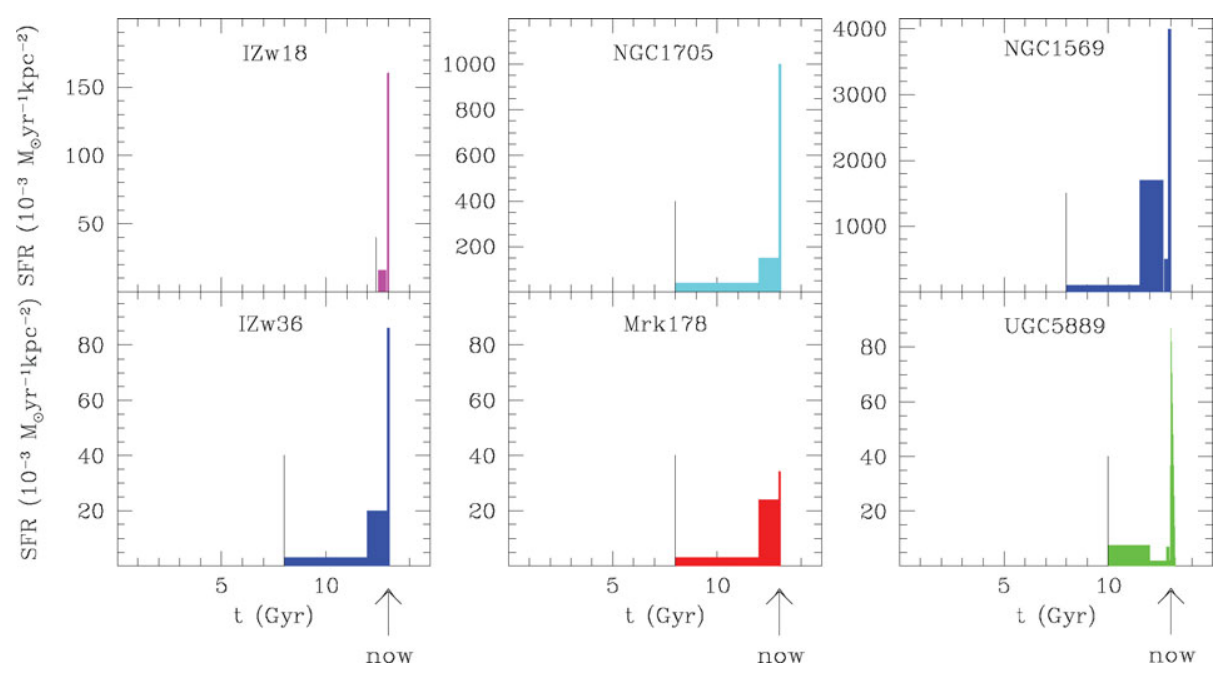

Figure 7. SFHs in late-type galaxies derived with the synthetic CMD method. In all panels the SF rate per unit area as a function of time is plotted. The thin vertical line in each panel indicates the reached lookback time. References: NGC 1569, Greggio et al. (1998), Angeretti et al. (2005); NGC 1705, Annibali et al. (2003); IZw18, Aloisi et al. (1999); IZw36, Schulte-Ladbeck et al. (2001); Mrk178, Schulte-Ladbeck et al. (2000); UGC 5889, Vallenari et al. (2005).

of the MW (SAB bc II-III) also shows a rather continuous SF regime, but with larger differences between the rate of the SF peaks and dips, see e.g. Rocha-Pinto \& Maciel (1997), Hernandez, Valls-Gabaud \& Gilmore (2000) and Cignoni et al. (2006). M33 (SA c III) definitely has significant bumps and gasps over its SFH, with a distribution of SFR with time almost indistinguishable from that derived for the late-type dwarf Leo A. One could then argue that the later the morphological type and the lower the luminosity class of the spirals, the more similar their SFH to those of late-type dwarfs.

Aside from speculations, the general results drawn from all the SFHs derived so far for galaxies with CMDs studies can be summarized as follows:

- Evidence of long interruptions in the SF activity is found only in early-type galaxies;

- Few early-type dwarfs have experienced only one episode of SF activity concentrated at the earliest epochs: many show instead extended or recurrent SF activity;

- No galaxy currently at its first SF episode has been found yet;

- No frequent evidence of strong SF bursts is found in late-type dwarfs;

- There is no significant difference in the SFH of dIrrs and BCDs, except for the current SFR.

Acknowledgements The Symposium organizers and IAU are gratefully acknowledged for partial financial support. I thank M. Cignoni and A. Cole for preparing figures ad hoc for this paper, and A. Dolphin and C. Gallart for the SFH plots shown in advance of publication. Some of the results described here have been obtained thanks to fruitful, recurrent and pleasant collaborations with A. Aloisi, L. Angeretti, F. Annibali, M. Cignoni, L. Greggio, A. Nota, and E. Sabbi.

\section{References}

Aloisi, A., Tosi, M., \& Greggio, L. 1999, AJ, 118, 302

Aloisi, A., Clementini, G., Tosi, M., Annibali, F., Contreras, R. et al. 2007, ApJ, 667, L151

Aloisi, A., van der Marel, R. P., Mack, J., Leitherer, C., Sirianni, M., \& Tosi, M. 2005, AJ, 631, L45 
Angeretti, L., Tosi, M., Greggio, L., Sabbi, E., Aloisi, A., \& Leitherer, C. 2005, AJ, 129, 2203 Annibali, F., Greggio, L., Tosi, M., Aloisi, A., \& Leitherer, C. 2003, AJ, 126, 2752

Aparicio, A. \& Gallart, C. 2004, AJ, 128, 1465

Barker, M. K., Sarajedini, A., Geisler, D., Harding, P., \& Schommer, R. 2007, AJ 133, 1138

Bertelli, G., Bressan, S., Chiosi, C., \& Nasi, E. 1994, A\&AS, 106, 275

Bertelli, G. \& Nasi, E. 2001, AJ, 121, 101

Brown, T. M. 2006, in The Local Group as Astrophysical Laboratory, M. Livio, T. M. Brown eds, STScI Symp. Ser. 17 (CUP), p.111

Brown, T. M., Beaton, R., Chiba, M., Ferguson, H. C., Gilbert, K. M. et al. 2008, ApJ, 658, L121

Chiosi, E., Vallenari, A., Held, E. V., Rizzi, L., \& Moretti, A. 2006, A\&A, 452, 179

Cignoni, M., Degl'Innocenti, S., Prada Moroni, P. G., \& Shore, S. N. 2006, A\&AA, 459, 783

Cignoni, M., Sabbi, E., Nota, A., Tosi, M., Degl'Innocenti, S., Prada Moroni, P., Angeretti, L., Carlson, L., Gallagher, J., Meixner, M., Sirianni, M., \& Smith, L. J. 2009, AJ, in press

Cole, A. A., Skillman, E. D., Tolstoy, E., Gallagher, J. S., Aparicio, A., et al. 2007, ApJ, 659, L17

Dohm-Palmer, R. C., Skillman, E. D., Gallagher, J. S., Tolstoy, E., Mateo, M., Dufour, R. J., Saha, A., Hoessel, J., \& Chiosi, C. 1998, AJ, 116, 1227

Dohm-Palmer, R. C., Skillman, E. D., Mateo, M., Saha, A., Dolphin, A., Tolstoy, E., Gallagher, J. S., \& Cole, A. A. 2002, AJ, 123, 813

Dolphin, A. E. 2002, MNRAS, 332, 91

Dolphin, A. E., Weisz, D. R., Skillman, E. D., \& Holtzman, J. A. 2005, astro-ph/0506430

Dolphin, A. E., Walker, A. R., Hodge, P. W., Mateo, M., Olszewski, W. W., Schommer, R. A., \& Suntzeff, N. B. 2001, ApJ, 562, 303

Fagotto, F., Bressan, A., Bertelli, G., \& Chiosi, C. 1994a, A\&AS, 104, 365

Fagotto, F., Bressan, A., Bertelli, G., \& Chiosi, C. 1994b, A\&AS, 105, 29

Ferraro, F. R., Fusi Pecci, F., Tosi, M., \& Buonanno, R. 1989, MNRAS, 241, 433

Gallart, C., Aparicio, A., Bertelli, G., \& Chiosi, C. 1996, AJ, 112, 1950

Greggio, L., Tosi, M., Clampin, M., De Marchi, G., Leitherer, C., Nota, A., \& Sirianni, M. 1998, ApJ, 504, 725

Harris, J. \& Zaritsky, D. 2004, ApJ, 127, 1531

Hernandez, X., Gilmore, G., \& Valls-Gabaud, D. 2000, MNRAS, 317, 831

Hernandez, X., Valls-Gabaud, D., \& Gilmore, G. 2000, MNRAS, 316, 605

Holtzman, J. A., Gallagher, J. S., Cole, A. A., Mould, J. R., et al. 1999, AJ, 118, 2262

Holtzman, J. A., Afonso, C., \& Dolphin, A. E. 2006, ApJS, 166, 534

Hurley-Keller, D., Mateo, M., \& Nemec, J. 1998, AJ, 115, 1840

Lynds, R., Tolstoy, E., O'Neil., E. J. Jr., \& Hunter, D. A. 1998, AJ, 116, 146

Marconi, G., Tosi, M., Greggio, L., \& Focardi, P. 1995, AJ, 109, 173

Noel, N. E. D., Gallart, C., Costa, E., \& Mendez, R. A. 2007, AJ, 133, 2037

Rejkuba, M., Greggio, L., \& Zoccali, M. 2004, A\&AS, 415, 915

Rocha-Pinto, H. J. \& Maciel, W. J. 1997, MNRAS, 289, 882

Seiden, P. E., Schulman, L. S., \& Gerola, H. 1979, ApJ, 232, 709

Schulte-Ladbeck, R. E., Hopp, U., Greggio, L., \& Crone, M. M. 2000, AJ, 120, 1713

Schulte-Ladbeck, R. E., Hopp, U., Greggio, L., Crone, M. M., \& Drozdovsky, I. O. 2001, AJ, 121,3007

Smecker-Hane, T. A., Cole, A. A., Gallagher, J. S. III., \& Stetson, P. B. 2002, ApJ, 566, 239

Smecker-Hane, T. A., Stetson, P. B., Hesser, J. E., \& Vandenberg, D. A. 1996, in From stars to galaxies, PASP Conf.Ser., 98, 328

Tolstoy, E. 1996, ApJ, 462, 684

Tosi, M., Greggio, L., Marconi, G., \& Focardi, P. 1991, AJ, 102, 951

Tosi, M., Sabbi, E., Bellazzini, M., Aloisi, A., Greggio, L., Leitherer, C., \& Montegriffo, P. 2001, $A J, 122,127$

Vallenari, A., Schmidtobreick, L., \& Bomans, D. J. 2005, A\&SAp, 435, 821

Young, L. M., Skillman, E. D., Weisz, D. R., \& Dolphin, A. E. 2007, ApJ, 659, 331 


\section{Discussion}

G. Meurer: Concerning the recent "gasp" in star formation seen in low-surfacebrightness dwarf galaxies, I don't think these can be attributed to the star formation history, but rather are induced by an under-populated upper end of the IMF. This is all explained in my poster (\#42).

M. Tosi: I have not myself worked on the LSBs. Could your results be attributed to stochastic effects on the IMF in very low-density environments, rather than to actual IMF variations?

R. WySE: I was very interested in your result that you always see stars as old as your limits. Could you say more about the prospects of pushing deeper, to see if it's like the Local Group, where all galaxies contain 10-12 Gyr old stars?

M. Tosi: It will not be easy to reach CMD phases (e.g., HBs) typically that old outside the Local Group in star-forming galaxies, because their young stars will always tend to hide the much fainter oldest stars. The best instrument for such a goal remains a space-based telescope.

J. Melbourne: In response to the question on Adaptive Optics, I do AO at Keck and I would agree that you will not do well with the very young stars but you might do very well on the AGB.

M. Tosi: Yes, AGBs will be very well measured with AO, but won't necessarily trace the oldest stellar populations.

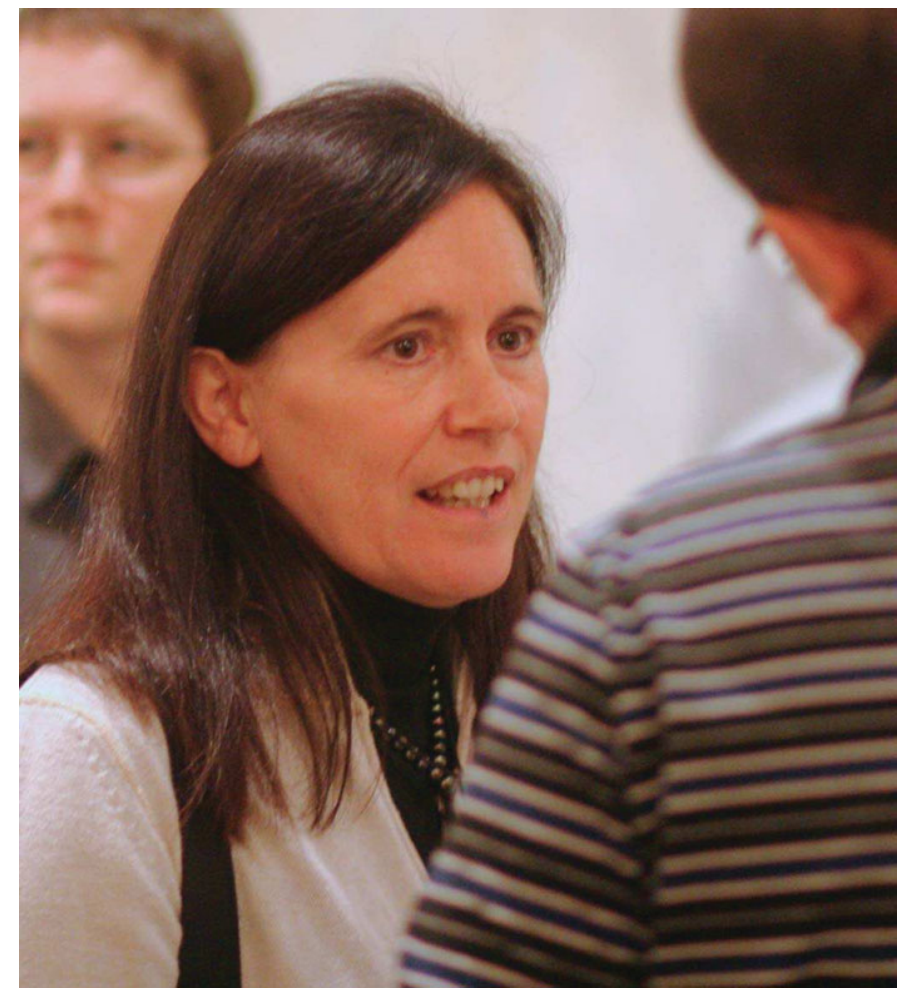

Monica Tosi 

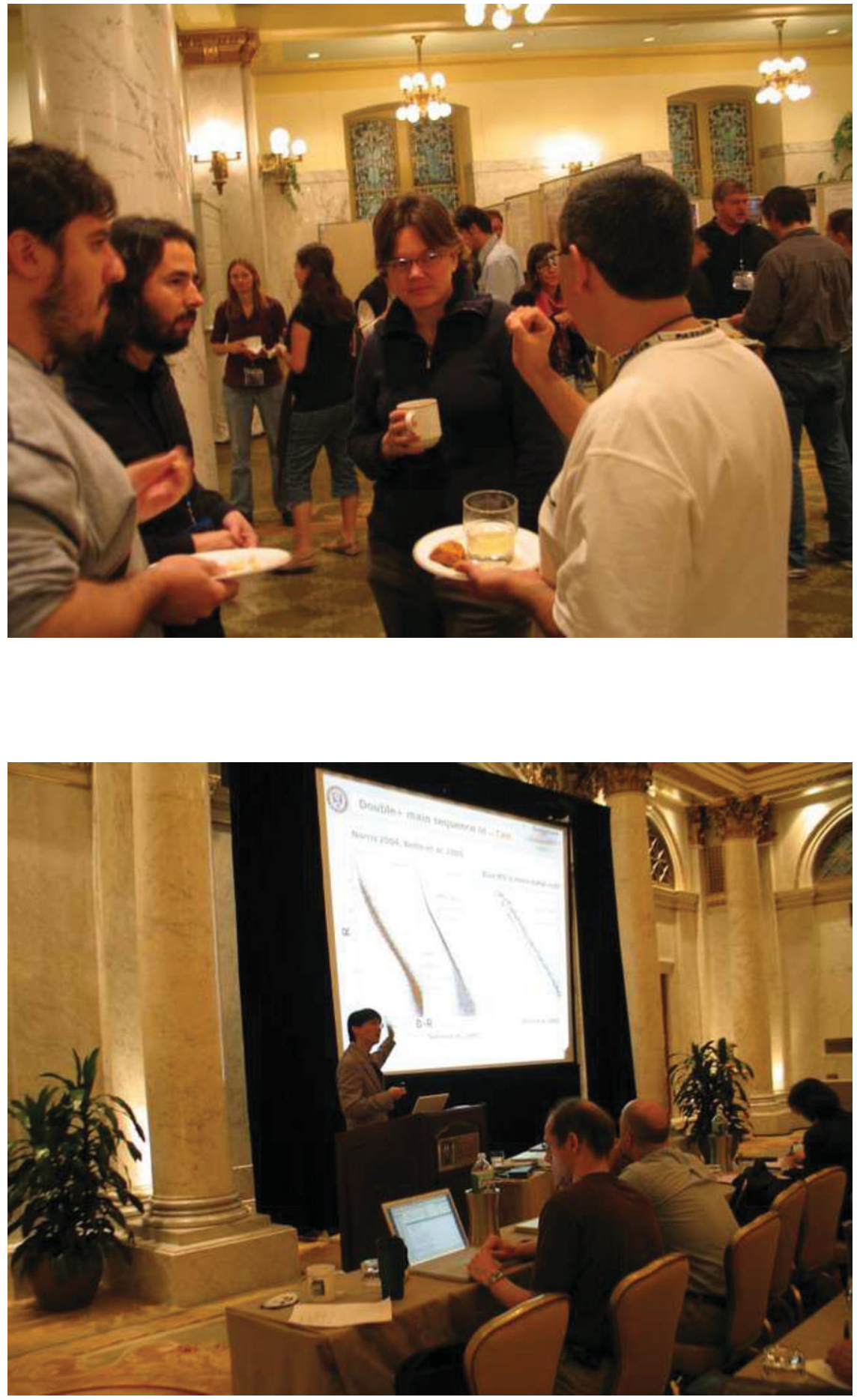